\title{
CONTRÔLE DE L'ACIDITÉ GASTRIQUE PAR LA CIMÉTIDINE DONNÉE EN PRÉMÉDICATION AVANT L'ANESTHÉSIE GÉNÉRALE: EFFICACITÉ ET TOLÉRANCE
}

\author{
D. Riche, Ch, Conselller, B. Coulbois, D. Ortega, \\ M-Ch. Terestchenko et J-M. Pacouret*
}

\begin{abstract}
RÉSUMÉ
La cimétidine administrée à la dose de $400 \mathrm{mg}$ par voie intramusculaire 60 minutes avant le début de l'anesthésie diminue l'acidité gastrique durant toute l'anesthésie générale, sans modifier significativement le volume des sécrétions. Elle diminue le pourcentage des sujets présentant un $\mathrm{pH}$ gastrique inférieur à 2,5 à l'induction et au réveil.

La cimétidine ne semble pas modifier le profil d'action des produits couramment utilisés en anesthésie. Elle n'entraîne pas de modifications significatives des principaux paramètres cardiovasculaires et électrocardiographiques.
\end{abstract}

Mots Clefs: Premedication, cimétidine; acidite Gastrique, controle, cimétidine.

LA CIMÉTIDINE † a fait la preuve de son efficacité en pathologie digestive. Il semblait donc logique de chercher à préciser dans quelle mesure elle pouvait être efficace en anesthésie générale pour alcaliniser le contenu gastrique, dans le but de diminuer le risque de survenue de pneumopathie chimique de déglutition. Ce risque d'inhalation de liquide gastrique persiste pendant toute la durée de l'anesthésie et au moment du réveil. Nous avons donc étudié la sécrétion gastrique durant toute l'anesthésie générale, après avoir administré la cimétidine par voie intramusculaire au moment de la prémédication.

Les études effectuées jusque là dans cette perspective $e^{7.8 .14 .16 .30 .32 .38 .44 .50}$ n'ont généralement pas porté de façon systématique sur la tolérance du produit au cours de l'anesthésie. Nous nous sommes donc attachés à rechercher d'éventuelles interférences avec les agents anesthésiques couramment utilisés.

\section{Patients et MÉthodes}

Cette étude a donc été faite en deux parties. Nous avons tout d'abord cherché à apprécier l'efficacité du produit sur une série de 84 patients de chirurgie orthopédique réglée. Dans un deuxième temps la tolérance du produit a été

\footnotetext{
* Département d'Anesthésie et de Réanimation Chirurgicale du C.H.U. Paris-Ouest. Hôpital Ambroise Pare. 9, A venue Ch. de Gaulle 92100-Boulogne/Seine France.

Tirés à part: Christian Conseiller - même adresse. $\nmid$ Tagamet $^{(0)}$ : Laboratoires Smith, Kline, French.
}

testée chez 100 patients de chirurgie générale réglée. L'étude a été menée en double aveugle et tirage au sort. Elle a été réalisée chez des adultes à l'exclusion des femmes enceintes ou allaitantes.

\section{Méthodes}

Tous les sujets, à jeun, ont été prémédiqués par voie intramusculaire, une heure avant le début de l'anesthésie, avec $100 \mathrm{mg}$ d'hydroxyzine et $0,5 \mathrm{mg}$ de sulfate d'atropine. Ils ont reçu au mème moment et par la même voie soit $400 \mathrm{mg}$ de cimétidine, soit du sérum physiologique, ce qui déterminait ultérieurement deux groupes: un groupe cimétidine et un groupe témoin. Une réinjection intraveineuse de $\mathbf{4 0 0} \mathrm{mg}$ de cimétidine ou de sérum physiologique était faite trois heures après l'induction lorsque l'intervention était de longue durée.

\section{a) Etude de l'efficacité}

Une sonde gastrique à double courant, type Salem, était mise en place permettant le recueil du liquide gastrique avant la prémédication, à l'induction, toutes les demi-heures au cours de l'anesthésie générale et au moment du réveil. Le volume des prélèvements était mesuré et le $\mathrm{pH}$ déterminé par une électrodeł à $\mathrm{pH}$. La position de la sonde était vérifiée par auscultation de l'épigastre, en injectant de l'air. A chaque fois qu'un recueil était négatif, la sonde était enlevée

¥G.297. $\mathrm{pH}$ mètre 62. Radiometer, Copenhague, Danemark.

Canad. Anaesth. Soc. J., vol. 28, no. 5, September 1981 
puis remise en place. L'induction était faite avec 4 à $6 \mathrm{mg} \cdot \mathrm{kg}^{-1}$ de thiopental et $1.5 \mathrm{mg} \cdot \mathrm{kg}^{-1}$ de succinyldicholine, ce qui permettait l'intubation orotrachéale pour tous les sujets. En plus d'un mélange d'oxygène et de protoxyde d'azote, l'entretien était assuré, soit en ventilation spontanée par halothane et dextromoramide, soit en ventilation contrôléc par phénopéridine et bromure de pancuronium.

\section{b) Etude de la tolérance}

Les patients étaient répartis en deux populations égales, l'une recevant une anesthésie à l'halothane en ventilation spontanée et l'autre une anesthésie comportant curare et morphinique en ventilation contrôlée. Dans les deux groupes l'induction anesthésique était faite avec thiopental et succinylcholine. L'anesthésie était entretenue avec protoxyde d'azote et oxygène en mélange à 66 pour cent. Tous les malades étaient sous surveillance électrocardioscopique continue pendant l'anesthésie. De plus un enregistrement électrocardiographique de plusieurs minutes était effectué avant l'induction, à l'intubation, pendant la réinjection, à l'extubation et au réveil. Le délai de réveil a été défini comme le temps écoulé entre l'arrêt du protoxyde d'azote et le contact verbal.

\section{Resultats}

Comme il s'agit d'une étude en double aveugle, nous avons vérifié rétrospectivement que pour les deux parties les deux groupes cimétidine et témoin sont comparables. Il n'apparait pas de différence significative dans la répartition des sexes, des âges, des durées d'anesthésie et des positions des patients sur la table d'opération pendant l'intervention chirurgicale. Ce dernier paramètre a été introduit pour tenir compte des difficultés de recueil du liquide gastrique éventuellement liées à la position. Il n'y a pas non plus de différence entre les deux groupes en ce qui concerne l'état secrétoire de l'estomac au moment de la prémédication. Les antécédents cardiaques sont aussi identiques pour les deux groupes de malade.

\section{a) Etude de l'efficacité}

Nous ne constatons pas de différence significative entre les deux groupes en ce qui concerne le volume des secrétions gastriques au cours de l'anesthésie générale (Figure 1). Cependant si nous considérons les sujets dont le recueil de liquide est négatif, une différence significative

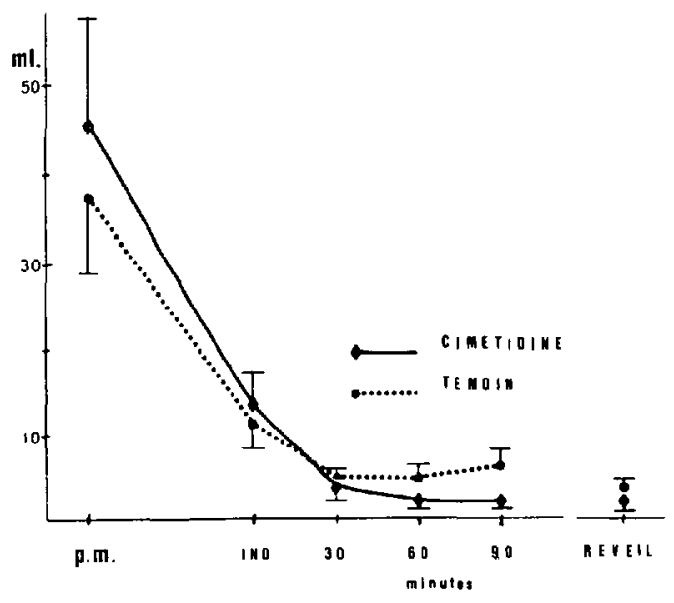

FIGURE 1 Volume de liquide gastrique recueilli au cours de l'anesthésie générale. $P M=$ prémédication. $\mathrm{IND}=$ induction, $\mathrm{REV}=$ réveil.

apparaît au moment du réveil puisque 40 pour cent des sujets du groupe cimétidine contre 17 pour cent des sujets du groupe témoin $(p<0,02)$ ont un recueil négatif à ce moment là.

Pour ce qui est de l'acidité gastrique, nous avons étudié les variations de la concentration moyenne en ions $\mathrm{H}^{+}$et en avons déduit les $\mathrm{pH}$ moyens $^{22-24}$ (Tableau I et Figure 2). Les deux groupes se comportent de façon distincte. Après 30 minutes d'anesthésie $\left[\mathrm{H}^{+}\right]$est en moyenne de $6,94 \times 10^{5} \mathrm{nmol} \cdot \mathrm{l}^{-1}(\mathrm{pH}=3,16)$ chez les malades ayant reçu de la cimétidine contre $5,42 \times 10^{6}$ nmol $\cdot l^{-1}(\mathrm{pH}=2,27)$ pour le groupe témoin $(\mathrm{p}<$ $0,01)$. Après 60 minutes d'anesthésie les valeurs respectives de $\left[\mathrm{H}^{+}\right]$sont $5 \times 10^{5}$ et $3,49 \times$ $10^{6} \mathrm{nmol} \cdot l^{-1}$. Au moment du réveil la différence est encore plus significative avec des valeurs de $1,68 \times 10^{\mathrm{s}} \mathrm{nmol} \cdot 1^{-1}(\mathrm{pH}=3,77)$ pour le groupe cimétidine et $7,7 \times 10^{6} \mathrm{nmol}^{-1} 1^{-1}(\mathrm{pH}=2,21)$ pour le groupe témoin $(\mathrm{p}<0,01)$.

Nous n'avons pas retrouvé de différence significative entre les deux groupes apres quatre vingt minutes d'anesthésie parce qu'il ne reste plus assez de sujets endormis à ce moment là, ce qui diminue la puissance des tests stastistiques.

La répartition de sujets présentant un $\mathrm{pH}$ gastrique inférieur à 2,5 est significativement différente entre les deux groupes lors de l'induction: 14,6 pour cent pour le groupe cimétidine contre 33,3 pour cent pour le groupe témoin $(\mathrm{p}<$ $0,05)$, à 30 minutes d'anesthésie: 9,7 pour cent pour le groupe cimétidine contre 35,5 pour cent pour le groupe témoin $(p<0,02)$ et au réveil: 0 pour cent pour le groupe cimétidine contre 34 pour cent pour le groupe témoin $(p<0,0015)$ (Figure 3). 
TABLEAU I

Comparaison des Contenus acides de L'Estomac au Cours de L'ANESTHÉSIE GÉNÉRALE

\begin{tabular}{|c|c|c|c|c|}
\hline & $\begin{array}{c}{\left[\mathrm{H}^{+}\right] \mathrm{nmol} \cdot \mathrm{l}^{-1}} \\
\text { moyenne } \pm \text { ecart type }\end{array}$ & & $\begin{array}{c}{\left[\mathrm{H}^{+}\right] \mathrm{nmol} \cdot \mathrm{l}^{-1}} \\
\text { valeurs extrèmes }\end{array}$ & $\underset{\text { moyen }}{\mathrm{pH}}$ \\
\hline $\begin{array}{l}\text { Avant prémed } \\
\text { cimétidine }\end{array}$ & cation $\begin{array}{r}9,50 \times 10^{6} \\
\pm 18,4 \times 10^{6}\end{array}$ & \multirow[b]{2}{*}{ NS } & $\begin{array}{r}7,9 \times 10^{7} \\
+2,0 \times 10^{2}\end{array}$ & 2,02 \\
\hline témoin & $\begin{array}{r}5,32 \times 10^{6} \\
\pm 14,4 \times 10^{6}\end{array}$ & & $\begin{array}{r}6,3 \times 10^{7} \\
\pm 3,2 \times 10\end{array}$ & 2,27 \\
\hline $\begin{array}{l}\text { Induction } \\
\text { cimétidine }\end{array}$ & $\begin{array}{r}2,37 \times 10^{6} \\
\pm 6,35 \times 10^{6}\end{array}$ & \multirow[b]{2}{*}{ NS } & $\begin{array}{r}3,2 \times 10^{7} \\
\pm 1,6 \times 10^{7}\end{array}$ & 2,63 \\
\hline témoin & $\begin{array}{r}5,73 \times 10^{6} \\
\pm 10,9 \times 10^{6}\end{array}$ & & $\begin{array}{r}5,0 \times 10^{7} \\
\pm 1,6 \times 10\end{array}$ & 2,34 \\
\hline $\begin{array}{l}\text { Induction + } 3 \\
\text { cimétidine }\end{array}$ & min $\begin{array}{r}6,94 \times 10^{5} \\
\pm 21,3 \times 10^{5}\end{array}$ & \multirow{2}{*}{$\dagger$} & $\begin{aligned} & 7,9 \times 10^{6} \\
\pm & 6,3\end{aligned}$ & 3,16 \\
\hline témoin & $\begin{array}{r}5,42 \times 10^{6} \\
\pm 9.10 \times 10^{6}\end{array}$ & & $\begin{array}{r}4,0 \times 10^{7} \\
\pm 2,0 \times 10\end{array}$ & 2,27 \\
\hline $\begin{array}{l}\text { Induction + } 6 \\
\text { cimétidine }\end{array}$ & $\begin{array}{r}\min \\
\pm, 40 \times 10^{5} \\
\pm 15,0 \times 10^{5}\end{array}$ & \multirow{2}{*}{ * } & $\begin{aligned} & 5,0 \times 10^{6} \\
\pm & 7,9\end{aligned}$ & 3,27 \\
\hline témoin & $\begin{array}{r}3,49 \times 10^{6} \\
\pm 5,90 \times 10^{6}\end{array}$ & & $\begin{aligned} & 3,2 \times 10^{7} \\
\pm & 7,9\end{aligned}$ & 2,46 \\
\hline $\begin{array}{l}\text { Induction }+9 \\
\text { cimétidine }\end{array}$ & min $\begin{array}{r}3,45 \times 10^{5} \\
\pm 7,20 \times 10^{5}\end{array}$ & \multirow{2}{*}{ NS } & $\begin{aligned} & 2.0 \times 10^{6} \\
\pm & 2.5 \times 10\end{aligned}$ & 3,45 \\
\hline témoin & $\begin{array}{r}1,22 \times 10^{7} \\
\pm 2,27 \times 10^{7}\end{array}$ & & $\begin{array}{r}6,3 \times 10^{7} \\
\pm 1,3 \times 10\end{array}$ & 1,91 \\
\hline $\begin{array}{l}\text { Réveil } \\
\text { cimétidine }\end{array}$ & $\begin{array}{r}1.68 \times 10^{5} \\
\pm 5,10 \times 10^{5}\end{array}$ & \multirow{2}{*}{$\dagger$} & $\begin{aligned} & 2,0 \times 10^{6} \\
\pm & 1,6 \times 10\end{aligned}$ & 3,77 \\
\hline témoin & $\begin{array}{r}7,70 \times 10^{6} \\
\pm 1,45 \times 10^{6}\end{array}$ & & 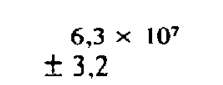 & 2,21 \\
\hline
\end{tabular}

$$
\begin{aligned}
& * P<0,02 \\
& \dagger P<0,01
\end{aligned}
$$

\section{b) Tolérance}

Il n'y a pas de différence significative en ce qui concerne la quantité de produits anesthésiques consommés dans le groupe cimétidine et le groupe placébo (Tableau II). La cimétidine ne paraît donc pas interférer avec la consommation des produits anesthésiques utilisés dans cette étude.

Le délai de réveil n'est pas différent dans les deux groupes $11 \pm 1$ min dans le groupe placébo, contre $11,5 \pm 0,9 \mathrm{~min}$ dans le groupe cimétidine. Par contre, le réveil a été considéré comme calme dans le groupe cimétidine 46 fois sur 49 , contre 36 fois sur $\mathbf{4 6}$ dans le groupe placébo. Cette diffërence est significative à cinq pour cent.
Les différents paramètres cardiovasculaires et électrocardiographiques ont été étudiés avec une attention particulière.

La cimétidine ne majore pas la fréquence des troubles du rythme à l'induction anesthésique. Les troubles du rythme sont identiques quant à leur type: extrasystoles ventriculaires (E.V.) ou supraventriculaires (E.S.V.), et quant à leur nombre total. Nous avons fait la même constatation à l'extubation (Tableau III). Les principaux paramètres électrocardiographiques espaces $P R$, QT et QRS, ont évolué de façon similaire sans différence significative quelque soit le type d'anesthésie. En ce qui concerne la fréquence cardiaque elle évolue de façon identique (Tableau 
RICHE, et al.: CONTRÔLE DE L'ACIDITÉ GASTRIQUE

TABLEAU II

Consommation de Produits Anesthésiques

\begin{tabular}{lcrc}
\hline \hline & Placebo & Cimétidine \\
\hline Thiopental $\mathrm{cg} \cdot \mathrm{kg}^{-1}$ & $0,823 \pm 0,19$ & $0,822 \pm 0,23$ & N.S. \\
Phenoperidine $\mathrm{mg} \cdot \mathrm{kg}^{-1} / \mathrm{mn}$ & $52,3 \times 10^{-5} \pm 18,8 \times 10^{-5}$ & $48 \times 10^{-5} \pm 18 \times 10^{-5}$ & N.S. \\
Bromure de pancuronium $\mathrm{mg} \cdot \mathrm{kg}^{-1} / \mathrm{mn}$ & $9,52 \times 10^{-4} \pm 4,4$ & $9,16 \times 10^{-4} \pm 3,74$ & N.S. \\
Halothane (répartition des concentrations) & & & \\
0,5 à 0,75 p. cent Nombres de malades & 7 & 17 & [N.S.] \\
là 1,5 p. cent & 16 & & \\
\hline
\end{tabular}

TABLEAU III

Fréquence des Troubles du Rythme à l'INduction ANESThÉsique ET Aं L'EXTUBATION

\begin{tabular}{lrc}
\hline \hline & $\begin{array}{c}\text { Placebo } \\
\mathrm{N}=99\end{array}$ & $\begin{array}{c}\text { Cimétidine } \\
\mathrm{N}=101\end{array}$ \\
\hline $\begin{array}{l}\text { Induction } \\
\quad \text { Extrasystoles supraventriculaires }\end{array}$ & 8 & 11 N.S. \\
$\quad \begin{array}{l}\text { Extrasystoles ventriculaires } \\
\text { Nombre total des malades ayant présenté des extrasystoles }\end{array}$ & 16 & 19 N.S. \\
Extubation & 24 N.S. \\
$\quad$ Extrasystoles supraventriculaires & 3 & 12 N.S. \\
$\quad$ Extrasystoles ventriculaires & 9 & 11 N.S. \\
Nombre total des malades ayant présenté des extrasystoles & 11 & 12 N.S. \\
\hline
\end{tabular}

Il n'y a aucune différence significative dans la fréquence des troubles du rythme suivant le type d'anesthésie.

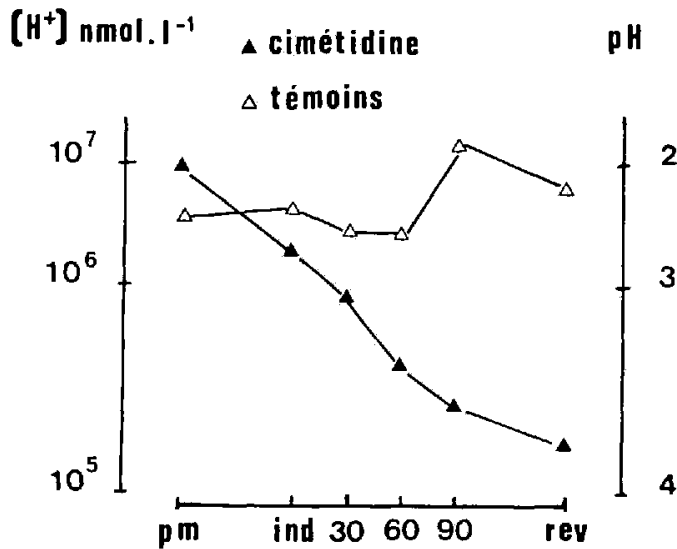

FIgure 2 Acidité du liquide gastrique au cours de l'anesthésie générale. $P M=$ prémédication. $I N D=$ induction. $\mathrm{REV}=$ réveil.

IV) dans les deux groupes de sujets et quelque soit le type de l'anesthésie; toutefois on remarque qu'au delà de la deuxième heure d'anesthésie la fréquence cardiaque semble plus lente chez les malades ayant reçu de la cimétidine. Cette différence n'est pas statiquement significative car peu de patients ont été endormis plus de deux heures.

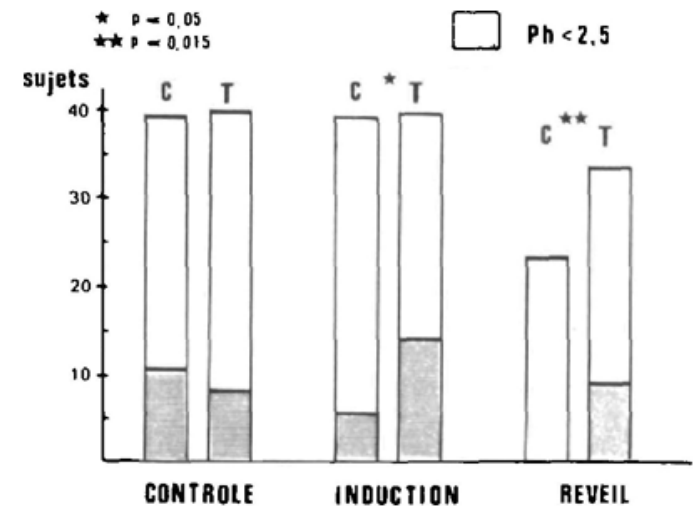

Figure $3 \mathrm{pH}$ gastrique inférieur à 2.5 chez les sujets ayant un recueil positif: répartition des sujets à l'induction et au réveil. $\mathrm{C}=$ cimétidine, $\mathrm{T}=$ témoins.

La cimétidine ne semble pas favoriser de modification des pressions artérielles systoliques et diastoliques puisqu'il n'y a aucune différence significative en ce qui concerne l'évolution de ces pressions d'un groupe à l'autre et quelque soit le type d'anesthésie (Tableau IV).

Lorsque l'anesthésie a été de longue durée, l'injection intraveineuse directe de $400 \mathrm{mg}$ de cimétidine chez 9 malades en cours d'anesthésie 


\section{TABLEAU IV}

\begin{tabular}{|c|c|c|c|c|c|}
\hline $\begin{array}{l}\text { Temps (en minute } \\
\text { à partir de l'inducti } \\
\text { anesthésique }\end{array}$ & & to & 160 & 1120 & 1180 \\
\hline $\begin{array}{l}\text { Fréquence cardiaque } \\
\text { (en minutes) }\end{array}$ & $\begin{array}{l}\mathrm{C} \\
\mathrm{P}\end{array}$ & $\begin{array}{l}82,3 \pm 8,7 \\
78,8 \pm 9,8\end{array}$ & $\begin{array}{r}80,6 \pm 9,2 \\
81 \pm 13,4\end{array}$ & $\begin{array}{l}72,7 \pm 11,3 \\
77,9 \pm 12,7\end{array}$ & $\begin{array}{l}71,7 \pm 8,6 \\
74,2 \pm 10,7\end{array}$ \\
\hline $\begin{array}{l}\text { P.A. systolique } \\
\text { moyenne (kPa) }\end{array}$ & $\begin{array}{l}\mathrm{C} \\
\mathrm{P}\end{array}$ & $\begin{array}{l}16,8 \pm 2,8 \\
17,1 \pm 2,5\end{array}$ & $\begin{array}{l}16,3 \pm 2,9 \\
16,5 \pm 3,2\end{array}$ & $\begin{array}{l}17,6 \pm 3,3 \\
17,5 \pm 4,1\end{array}$ & $\begin{array}{l}17,8 \pm 2,5 \\
16,7 \pm 3,8\end{array}$ \\
\hline $\begin{array}{l}\text { P.A. diastolique } \\
\text { moyenne }(\mathrm{kPa})\end{array}$ & $\begin{array}{l}\mathrm{C} \\
\mathbf{P}\end{array}$ & $\begin{array}{l}10,5 \pm 1,6 \\
10,7 \pm 1,4\end{array}$ & $\begin{array}{l}10,6 \pm 1,6 \\
10,6 \pm 1,7\end{array}$ & $\begin{array}{l}11,8 \pm 2,1 \\
10,9 \pm 2,2\end{array}$ & $\begin{array}{l}11,2 \pm 1,3 \\
11,3 \pm 2,5\end{array}$ \\
\hline
\end{tabular}

Il n'y a aucune différence significative entre le groupe placebo ct le groupe prémédiqué par la Cimétidine.

(trois heures après induction), comparée en double aveugle à huit injections placébo, n'entraine aucune modification des principaux paramètres étudiés.

\section{Discussion}

La survenue d'une pneumopathie chimique de déglutition est essentiellement liée à l'acidité du liquide gastrique inhalé. Un pH inférieur à 2.5 est un facteur déterminant comme l'ont montré les travaux de Teabeaut en $1952^{48}$ et de Taylor et Pryse-Davies en 1966..6 Dans notre étude, 36 pour cent des sujets témoins à jeun ont un pH gastrique inférieur à 2,5 au moment de l'induction. Ce chiffre atteint 60 à 80 pour cent des sujets témoins dans certaines études anglosaxonnes. ${ }^{5,8.14,16,30,32,38,44,50}$ Lors du réveil, 34 pour cent des sujets témoins de notre étude ont un $\mathrm{pH}$ gastrique inférieur à 2,5 . Un pourcentage important de sujets est donc exposé au risque de pneumopathie chimique de déglutition à l'induction et au réveil lorsqu'il y a intubation trachéale. Le risque d'inhalation est encore plus grand en l'absence d'intubation trachéale et Culver a montré en $195 !^{10}$ qu'un sujet sur six inhale du liquide gastrique lors de l'anesthésie au masque. L'inhalation du liquide gastrique est responsable de la moitié des décès maternels en anesthésie obstétricale ${ }^{9}$ et de 11 à 23 pour cent des décès liés à l'anesthésie non obstétricale. ${ }^{1.5,15,17}$ C'est donc une complication grave que l'on s'est efforcé de prévenir par différents moyens et en particulier par l'utilisation des antiacides et des médications anticholinergiques. Les antiacides (trisilicate de magnésium, hydroxyde d'aluminium, citrate de sodium) ont cependant des effets inconstants. Leur efficacité diminue avec le temps, ${ }^{46}$ ils augmentent le contenu gastrique ${ }^{45}$ modifient de façon inconstante le $\mathrm{pH}^{27}$ et retardent l'évacuation gastrique. ${ }^{29}$ Ils peuvent eux même être à l'origine de pneumopathie chimique en cas d'inhalation dont la gravité serait comparable à celle des pneumopathies chimiques par inhalation acide. ${ }^{3,21,26,47}$ Les médications anticholinergiques ont un effet inconstant sur les modifications du $\mathrm{pH}$. Le caractère partiel de leur effet a été constaté chez les sujets témoins de notre étude puisque ils avaient tous reçu de l'atropine en prémédication. De plus, les anticholinergiques retardent la vidange gastrique et réduisent le tonus du sphincter inférieur de l'œesophage. ${ }^{40} \mathrm{Le}$ glycopyrolate, anticholinergique à radical ammonium quaternaire n'affecte pas la vidange gastrique $^{51}$ mais ses effets sur le $\mathrm{pH}$ gastrique sont tres variables ${ }^{2.45}$ et son efficacité semble nettement inférieure à celle de la cimétidine.$^{32}$

On s'est donc orienté vers la cimétidine, antagoniste des récepteurs $\mathrm{H}_{2}$ de l'histamine, s'opposant à la sécrétion acide de l'estomac déclenchée par I'histamine, l'acétylcholine et la gastrine, sans affecter la cinétique de la vidange gastrique ni le tonus du sphincter inférieur de I'nesophage. ${ }^{19}$

Il apparait dans notre étude que durant l'anesthésie générale l'apport de cimétidine en prémédication ne modifie pas significativement le volume des sécrétions gastriques par rapport à la prémédication traditionnelle par l'atropine. On sait que la cimétidine ne semble pas agir sur le volume des sécrétions basales, ${ }^{19}$ c'est probablement l'une des raisons de cette absence de différence significative sur le volume recuelli dans les deux groupes de malades. Par contre. il est interessant de noter qu'au réveil l'efficacité de l'adjonction de cimétidine apparait significative puisque 40 pour cent des malades du groupe 
cimétidine peuvent être considérés comme ayant l'estomac vide contre 17 pour cent seulement dans le groupe témoin. On peut se demander si la différence observée n'est pas due aussi au fait qu'au moment du réveil l'efficacité propre de l'atropine devient plus faible alors que la cimétidine est encore efficace à ce moment là, comme en témoigne son action sur le $\mathrm{pH}$.

L'administration de cimétidine par voie intramusculaire une heure avant l'induction, diminue significativement l'acidité gastrique trente minutes après le début de l'anesthésie, ce qui devra conduire à administrer ce produit quatre vingt dix minutes avant l'induction. Aux moments les plus exposés que sont l'induction et le réveil la cimétidine diminue de façon significative le pourcentage des sujets présentant un $\mathrm{pH}$ gastrique inférieur à 2.5.

Par ailleurs la cimétidine n'interfère pratiquement pas avec l'anesthésie générale. Bien que pouvant potentialiser l'action de certains produits dont le métabolisme est hépatique comme les anticoagulants oraux $x^{43}$ et le diazépam ${ }^{33}$, elle ne semble pas modifier le profil d'action du bromure de pancuronium, de la phénopéridine, du thiopental sodique et de l'halothane. Cependant la meilleure sédation que nous avons observée au réveil pourrait relever de ce mécanisme.

La cimétidine n'entraine pas de modification sensible des principaux paramètres de surveillance cardiovasculaire usuels. Elle est sans influence sur la fréquence des principaux troubles du rythme observés aux différents temps anesthésiques.

Cependant nous avons observe chez les patients endormis plus de deux heures une tendance à la bradycardie dans le groupe recevant de la cimétidine. Ceci est difficilement interprétable car la population est trop réduite au delà de deux heures d'anesthésie. Si la pratique de la prémédication par la cimétidine devait se généraliser, il serait important d'observer sur un plus grand nombre de sujets, au cours de l'anesthésie générale, l'évolution de la fréquence cardiaque trois heures après l'administration de ce produit; en effet plusieurs accidents ont été rapportés dans la littérature en dehors de l'anesthésie générale. Il s'agissait soit d'accidents bénins de bradycardie sinusale ou jonctionnelle et d'hypotension réversibles à l'arrêt du traitement et reproductibles après une nouvelle administration de cimétidine, ${ }^{31.36 .41}$ soit d'accidents plus graves de bradycardie sévère, de dissociation auriculo-ventriculaire, d'asystolie chez des su- jets déjà en état hémodynamique précaire à qui étaient administré de façon répétée. par voie intraveineuse directe, de fortes doses de cimétidine. ${ }^{4.6 .37}$ On sait en effet qu'il existe au niveau du cœur deux types de récepteurs histaminiques: les récepteurs $H_{1}$ développant une activité inotrope négative et les récepteurs $\mathrm{H}_{2}$ qui développent une activité inotrope et chronotrope positives. Zavecz et Levi ${ }^{52}$ ont montré sur le cour de porc que le blocage des récepteurs $\mathrm{H}_{2}$ par la cimétidine démasquait l'action inotrope négative de l'histamine liée à l'activation des récepteurs $\mathrm{H}_{1}$. Cette situation pourrait se voir chez l'homme lorsque les concentrations d'histamine et de cimétidine sont élevées au niveau du cour. Ceci serait réalisé chez les sujets en état d'agression'1,20.28.35.39 recevant de fortes doses de cimétidine par voie intraveineuse, et à plus forte raison s'ils sont en insuffisance rénale. ${ }^{13,19,25}$ En dehors de ces états d'agression et chez les sujets non anesthésiés, aucune étude prospective n'a mis en évidence de tels effets cardiaques. La cimétidine n'influence pas le débit cardiaque, la fréquence cardiaque, la pression artérielle ${ }^{12,42}$ et la réponse du cœur à l'effort avec ou sans propanolol. ${ }^{49}$ Elle ne modifie pas la fonction du nœud sinusal. ${ }^{18}$ Il lui aurait été trouvé, en dehors de l'anesthésie des propriétés anti-arythmisantes. ${ }^{34}$ Ces constatations tendent à prouver qu'en situation habituclle, l'histamine ne joue qu'un rôle négligeable sur la fonction cardiaque.

Il résulte de ces constatations et de notre étude que la cimétidine ne semble pas faire courir de risque cardiaque au cours de l'anesthésie générale.

\section{CONCLUSION}

La cimétidine administrée par voie intramusculaire quatre vingt dix minutes avant l'induction devrait présenter un grand intérêt dans la prévention des pneumopathies chimiques de déglutition secondaires à l'inhalation de liquide gastrique acide au cours de l'anesthésie générale. Nous pensons qu'elle est particulièrement indiquée chaque fois qu'une anesthésie générale est conduite au masque. Ce travail devrait susciter une étude analogue en anesthésie obstétricale.

La tolérance de la cimétidine donnée en prémédication nous a paru très bonne. Le produit n'interfère pas avec l'activité des agents anesthésiques employés dans cette étude. Ce travail ne fait pas apparaitre de contre-indication à l'utilisation de la cimétidine en prémédication en chirurgie réglée chez l'adulte et en dehors de la grossesse. 


\section{REMERCIEMENTS}

Ce travail à été effectué dans le service d'orthopédie (Pr. J-O; Ramadier) et le service de chirurgie générale (Pr. J.-C. Patel) de l'Hôpital A. Pare.

\section{BIBLIOGRAPHIE}

1. Bannister, W.K. \& Satillaro, A. J. Vomiting and aspiration during anesthesia. Anesthesiology, $23,251-264$ (1962).

2. Baraka, A., SaAb, M., Salem, M.R. \& Winnie, A.P. Control of gastric acidity by glycopyrrolate premedication in the parturient. Anesth. Anal., 56 , 642-645 (1977).

3. Bond, V.K., Soelting, R.K. \& Gupta, C.D. Pulmonary aspiration syndrome after inhalation of gastric fluid containing antacids. Anesthesiology, $51,452-453$ (1979)

4. Bournerias, F., Ganeval, D. \& Danan, G. Trouble du rythme cardiaque mortel au cours d'un traitement par la cimétidine. Nouv. Presse Med., 7 , 2069 (1978).

5. Clifton, B.S. \& Hotren, W.I.T. Deaths associated with anaesthesia. Brit. J. Anaesth.. 35, 250 259 (1963).

6. Cohen, J., Weetman, A.P., Dargie, H.D. \& KRIKLER, D.M. Life-threatening arrhythmias and intravenous cimetidine. Brit. Med. J., 2, 768(1979).

7. Coomes, D.W., Hooper, D. \& Colton, T. Preanesthetic cimetidine alteration of gastric fluid volume and pH. Anesth. Anal., 58, 183-188 (1979).

8. CoOmbs, D.W., Hooper, D. \& Colton, T. Acid aspiration prophylaxis by use of preoperatory oral administration of cimetidine. Anesthesiology, 51 . 352-356 (1979)

9. CRAWFORd J.S. The anesthetist's contribution to maternal mortality. Brit. J. Anaesth., 42, 70-73 (1970).

10. Culver, G.A., Makel, H.P. \& Beecher, H.K. Frequency of aspiration of gastric contents by the lungs during anesthesia and surgery. Ann. Surg., 133, 289-292 (1951)

11. Deforest, J.M. \& Hollis, Th.M. Shear stress and aortic histamine synthesis. Am. J. Physiol., 234, H. 701-705 (1978).

12. Delaney, J.P., Michel, H.M. \& Bond, J.P. Cimetidine and gastric blood flow. Surgery, 84 190-193 (1978)

13. Delchier, J.C., Soule, J.C. \& Bader, J.P. La cimetidine: Pharmacologie et toxicité. Nouvelle Presse. Med. . 7, 1735-1739 (1978).

14. Detmer, M.D., Pandit, S.K. \& Cohen, P.J Prophylactic single dose oral antacid therapy in the preoperative period comparison of cimetidine and Maalox ${ }^{\circledast}$. Anesthesiology, 51, 270-273 (1979).

15. DinNick, O.P. Deaths associated with anaesthesia. Observations on 600 cases. Anaesthesia, 19, 536556 (1964).

16. DobB, G., Jordan, M.J. \& Williams, J.G. Cimetidine in the prevention of the pulmonary acid aspiration (Mendelson's) Syndrome. Brit. J. Anaesth. 5I, 1-4 (1979).

17. Edwards, G., Morton, H.J.V., Pask, E.A. \&
W YLIE, W.D. Deaths associated with anaesthesia: a report of 1000 cases. Anaesthesia, 11, 194-220 (1956).

18. ENGEL, T.R. \& LuCK, J.C. Histamine 2 receptor antagonism by cimetidine and sinus node function. N. Engl. J. Med., 301, 591-592 (1979).

19. Finkelstein, W. \& Isselbacher, K.J. Cimetidine. N. Engl. J. Med. 299, 992-996 (1978).

20. Fischer, M.W. Lorenz, W., Neugebauer, E. \& Schmal, A. Histamine release and pathogenesis of stress ulcer in polytraumatized patients: a prospective randomized study on prophylactic effect of cimetidine. Zeitblatt Chir, 104, 810 (1979).

21. Gibrs, C.P., Schwarts, D.J., WynNe, J.W., HooD, C.I. \& KuCK, E.J. Antacid pulmonary aspiration in the dog. Anesthesiology, 51, 380-385 (1979).

22. Giesecke, A.H., Beyer, C.W. \& Kallus, F.T. More on interpretation of $\mathrm{pH}$ data. Anesth. Anal., 57, 379-381 (1978).

23. GiEsECKE, A.H. Averaging values for gastric $\mathrm{pH}$ incorrect. Anesthesiology, 50, 70-71 (1979).

24. Giesecke, A.H. Averaging $\mathrm{pH}$ vs $\mathrm{H}^{+}$values. Anesthesiology, 51, 481-483 (1979).

25. Grave, W., NadorP, J.H.S.M. \& Rutten, J.J.M.H. Cimetidine and renal failure. Lancet., $i i$, 719-720 (1977)

26. Heaney, G.A. \& Jones, H.D. Aspiration Syndromes in pregnancy. Brit. J. Anaesth., 51, 266$267(1979)$.

27. Hester, J.B. \& Heath, M.L. Pulmonary acid aspiration Syndrome: Should prophylaxis be routine? Brit. J. Anaesth. 49, 595-599 (1977).

28. Hirasuna, J.D., Shelub, I. \& Bolt, R.J. Hyperhistaminemia and peptic ulcer. West J. Med. 13I, $140-143$ (1979).

29. Hurwitz, A., Robinson, R.G. \& VATS, T.S. Effects of antacids on gastric emptying. Gastroenterology, 7I, 268-273 (1976).

30. HUSEMEYER, R.P., DAVENPORT, H.T. \& RAJASEKARAN, T. Cimetidine as a single oral dose for prophylaxis against Mendelson's Syndrome. Anaesthesia, 33, 775-778 (1978).

31. JefFerys, D.B. \& Vale, J.A. Cimetidine and bradycardia. Lancet $i, 828$ (1978).

32. Keating. P.J., Black, J.F. \& Waston, D.W Effects of glycopyrrolate and cimetidine on gastric volume and acidity in patients awaiting surgery. Brit. J. Anaesth., 50, 1247-1250 (1978).

33. Klotz, U. \& ReimanN, I. Delayed clearance of diazepam due to cimetidine. N. Engl. J. Med., 302 , 1012-1014 (1980).

34. Ligumsky, M., Shochina, M. \& RaChMILEWITZ, $D$. Cimetidine and arrhythmia suppression. Ann. Intern. Med., 89, 1008-1009 (1978).

35. Lorenz, W., Doenicke, A., Neugebauer, E. Schwarz, B., Schmal, A.\& Schoning, B. Definition of histamine release in human subjects and experimental animals using plasma histamine determination in the whole individual. Agents and Actions, 9, 35 (1979).

36. Luciano, J.J., Theodorov-Touchais, A.M. \& Souteyrand, P. Cimetidine: Trouble du rythme cardiaque au cours d'un traitement. Nouv. Presse Med., 7, 4049(1978).

37. MahoN, W.A. \& Kolton, M. Hypotension after intravenous cimetidine. Lancet, $i, 828$ (1978). 
38. Maliniak, K. "\& Vakil A.H. Pre-anesthetic cimetidine and gastric pH. Anesth. Anal., 58, 309-313 (1979).

39. Maura, G., Vaccari, A. \& Timiras, P.S. Effects of chronic stress on the development of histamine enzymes. Agents and Actions, 7, 437-442 (1977).

40. Misiewicz, J.J. Symposium on gastroesophageal reflux and its complications. Gut., 14, 233-237 (1973).

41. Reding, P., Devroede, C. \& Barbier, P. Bradycardia after cimetidine. Lancet, ii, (1977).

42. Samuel, I.O.\& DundeE, J.W. Influence of cimetidine on cardio-vascular parameters in man. J. $R$. Sop. Med., 72, 898-901 (1979).

43. Serlin, M.J. \& Williams, J.R.B. Cimetidine interaction with oral anticoagulants in man. Lancet, ii, 317-319 (1979).

44. Stoelting, R.K. Gastric fluid pH in patients receiving cimetidine. Anesth. Anal., 57, 675-677 (1978).

45. StoElting, R.K. Responses to atropine, glycopyrrolate and Riopan of gastric fluid $\mathrm{pH}$ and volume in adult patients. Anesthesiology, 48, 367-369 (1978).

46. TAyloR, G. \& Pryse-Davies, J. The prophylactic use of antacids in the prevention of the acid pulmonary aspiration syndrome (Mendelson's Syndrome). Lancet, $i, 288-291$ (1966).

47. TAYLOR, G. Acid pulmonary aspiration syndrome after antacids. Brit. J. Anaesth., 47, 615-616 (1975).

48. Teabaut, J.R. Aspiration of gastric contents: an experimental study. Am. J. Pathol., 28, 51-67 (1952).

49. Wartburton, Opie, L.H., Kennelly, B.M. \& MULler, F.O. Does cimetidine alter the cardiac response to exercise and propranolol? S. Afr. Med. J.55, 1125-1127(1979).

50. Weber, L.A. \& Hikschman, C.A. Cimetidine for prophylaxis of aspiration pneumonitis: Comparison of intramuscular and oral dosage schedules. Anesthesiology, 5I, $\$ 180$ (1979).

51. Young, R. \& Sun, D.C. Effect of glycopyrrolate on antral mobility, gastric emptying and intestinal transit. Ann. N.Y. Acad. Sci., 99, 174-178 (1962).

52. ZAVECZ, J.H. \& LEVI, R. Histamine-induced negative inotropism: mediation by $\mathrm{H}_{1}$ receptors. J. Pharmacol. Exp. Ther., 206, 274-280 (1978).

\section{Abstract}

Cimetidine $400 \mathrm{mg}$ was administered intramuscularly 60 minutes before the beginning of general anaesthesia. The double blind experiment was conducted on 84 patients divided in two groups: cimetidine and control. There was no difference in gastric fluid volume bet ween the two groups during general anaesthesia, but acid secretion decreased significantly in the cimetidine group. Values of $\mathrm{pH}$ lower than 2.5 were observed in 33.3 per cent at induction and 34 per cent at recovery in the control group against 14.6 per cent and 0 per cent in the cimetidine group.

Clinical tolerance to cimetidine was studied in 100 patients during operation. Cimetidine did not alter pharmacological action of usual anaesthetics. There were no significant changes in cardiovascular and electrocardiographic data. 